\title{
Single Image Defogging Based On Improved Dark Channel Priority
}

\author{
Hua JIANG \& Yong jun XU \\ Sichuan University of Science \& Engineering Dept. of Electronic Engineering, Zigong, Sichuan
}

\begin{abstract}
Though the method of dark channel priority has made meaningful results in the process of single image defogging, it has not worked well in the treatment of the gray area in images. The channel image is related to the transmission distribution rate and concentration of fog. This paper proposed an image defogging method based on the Peak Signal to Noise Ratio(PSNR) and rule of the dark channel priority. The experiments show that the detail of result image using this method is more distinct, and the processing efficient of gray area is better comparing with the original method.
\end{abstract}

KEYWORD: Dark channel priority; Single image; Image defogging; Peak Signal to Noise Ratio

\section{GENERAL INSTRUCTIONS}

It often lead to the quality of image captured by outdoor surveillance equipment degraded in the rain and fog weather conditions, mainly for poor contrast, low visibility and colors $\operatorname{dim}$ and so on. It has seriously affected the image of traffic navigation and target identification and extraction, and greatly reduces the performance of outdoor surveillance equipment. Therefore, the image restoration is a research topic of great significance for the image under the rain, fog and other inclement weather conditions.

At present, the research on the image defogging can be mainly divided into two categories. The first category is image enhancement method. The second is method of image defogging based on the physical model of image degradation. The processing of the former is mainly subjective, while the latter is mainly through the use of the degradation phenomenon of priority knowledge to rebuild a degraded image. The typical image enhancement algorithms to defogging include histogram equalization method[1-3], retinex theory method [4$6]$, and the method of combining the theory of wavelet transform. Histogram equalization algorithm is simple, the atomization image contrast can be improved to some extent, however, this method will result in the loss of gray levels, and the contrast is low, the depth information for a variety of atomization image, which the method becomes powerless. Therefore, Zhan Xiang and others, who judge by calculating image local standard deviation to judge the local image enhancement degree, and then stretch the atomization gray level image contrast enhancement local variance [4], but this method when it is calculating the local variance, it is selected of appropriate size sliding window, sliding window size is determined according to the degree of the atomization of image to artificial, therefore it can not meet the real-time requirements of the occasion. RUI YIBIN and others who combined Retinex theory based on the atomization characteristics of the image histogram, using multiscale Retinex (MSR) and the normal method of stretching interception atomization image processing [6], this method of treatment has made over the mist good results, however, MSR algorithm requires the processing result of the weighted average of the plurality of scales, and to a large number of convolution operators, making the process timeconsuming. Ma YUNFEI and others who by determining image contrast threshold function was established and combined the idea of wavelet transform to enhance image atomizing[7], because this method requires human judgment whether the sky area need to be addressed, so that the operation is more complex, and to split the sky. When using Gaussian filtering approach also makes losing gray level information. Defogging method using the physical model of image degradation mainly based on atmospheric modulation function, such as literature [8] and [9]. Application of atmospheric modulation transfer function mentioned to recover weather degraded images, but these methods require many a priority knowledge, such as: the right time, 
temperature, relative humidity, wind speed, atmospheric scattering and absorption coefficient, which is often difficult to obtain accurate data, resulting in increased error sources. Defogging of images captured in different angles [10-11] has made certain progress, but it is difficult to obtain a suitable image of ordinary photographer. He and others[12] who propose the method of dark channel priority to defog, this method can get better effect, but it is ineffective for processing image gray area.

According to the above problem, this paper proposed an improved single image defogging method based on the statistical regularity of the dark channel priority. Set the initial value of the concentration coefficient atomization of the image, and increase the concentration coefficient by a given step, get a group of images after defogging with these concentration coefficient, calculate the transmission distribution rate of fog based on peak signal to noise ratio (PSNR) of image before and after defogging, and thus more accurately estimate the concentration of fog, finally get a clearer picture of the recovery from the physical model of the atomization.

\section{THE PRINCIPLE OF THE DARK CHANNEL PRIORITY}

The method of dark channel priority to go after the image defogging is described as:

$$
I(x)=J(x) t(x)+A(1-t(x))
$$

Where, $\mathrm{I}(\mathrm{x})$ is the image of atomization, $\mathrm{t}(\mathrm{x})$ is the transmission distribution rate of the haze sky, A is the value of the luminance of the sky, the image may be dark channel strike. $\mathrm{J}$ (x) is the image after defogging. So to the process of defogging is a simple equation to solve the above.

The theory of the dark channel priority through the statistical regularity of a large number of outdoor natural images drawn: outdoor natural scene in the image of the three color channels of at least one channel color value is lower, it will become a lower after dark channel priority deal with, but if there is more brightness and high pixels, then consider these brightness from the sky haze. Dark channel image can be defined as:

$$
\operatorname{dark}_{-} I=\min \left(\min I^{c}(x)\right)
$$

dark_I $c \in\{R, G, B\}$ Dark channel image

$x \in \alpha(x)$ Sky brightness $\mathrm{A}$, and the initial transmission distribution rate $\mathrm{t}_{0}(\mathrm{x})$ can be obtained from the dark channel images Since t the theory of dark channel adopted the idea of local blocking, thus makes the initial transmission distribution rate exist obvious squares. So He and others [12] by Levin and others the Matting Laplacian matrix [13] the initial transmission graph optimization function to get the following value:

$$
E(t)=\lambda\left\|t_{0}-t\right\|^{2}+t^{T} L t,
$$

Convert the quadratic function to solve the linear system of equations, to calculate the optimized transmission figure $\mathrm{t}(\mathrm{x})$, so we can calculate the atomization image $\mathrm{J}(\mathrm{x})$.

\section{IMPROVED METHOD OF DARK CHANNEL PRIORITY FOR SINGLE IMAGE}

\subsection{Algorithm principle}

The Peak Signal to Noise Ratio (PSNR) of image is main reference quantity to estimate image quality [14], it can be described as:

$$
P S N R=20 \log _{10}\left(\frac{255}{R M S E}\right)
$$

where RMSE as standard deviation, And,

$$
R M S E=\sqrt{\frac{\sum[I(i, j)-J(i, j)]}{M * N}}
$$

Where $I(I, j)$ is the atomization image, $j(I, j)$ is the image after dealing with the dark channel. M, N is the size of the image. By the literature [12] and dark channel image formula (2), the brightness of the sky can be described as:

$$
A=\max \left(\max \left(\text { dark_I }_{-}\right)\right)
$$

The transmission distribution ratio of fog in the sky can be described as:

$$
t(x)=1-\omega \times\left(\frac{\text { dark_I }_{-}}{A}\right)
$$

In the formula $\omega_{\text {is concentration coefficient for }}$ atomization in the image, and $\omega \in(0,1)$. In this paper we set the initial value of the concentration coefficient of haze, then increase $\omega$ by the given step, from the formula (2), (4), (5), (1) we can get a set of defogging image $\mathrm{J}(\mathrm{x})$, then using the formula (3) a group of signal to noise ratio are obtained with the increased $\omega$. If the increase of peak signal to noise ratio is less than 0.01 or begins to decrease, then stops increasing $\omega$. And at this time can be considered the best of the atomization image concentration. Repeat the above steps, we get defogged image corresponding to the best estimate output of the fog concentration $\omega$. Finally, as a compare, calculate the average gradient of the defogged images obtained from the algorithm in this paper and that from literature [7], we find that the 
average gradient of defogged image using our method is bigger. The average gradient is clear degree of image, and it reflects the image detail [8]. Therefore, the detail of result image using this method is more distinct, and the processing efficient of gray area is better.

\subsection{Algorithm steps}

(1) Calculate the dark channel image dark $_{-} I$ for atomizing image $\mathrm{I}(\mathrm{x})$ by the formula:

$$
\operatorname{dark}_{-} I=\min \left(\min I^{c}(x)\right)
$$

(2) Calculate the sky brightness value using the formula;

$$
A=\max \left(\max \left(\operatorname{dark}_{-} I\right)\right)
$$

(3) Set initial value of haze concentration $\omega$ in the atomization image, with increase of $\omega$, the concentration of the haze will also increase, and $\omega \in(0,1)$;

(4) Calculating the transmission distribution rate $t(x)$ of haze in the sky by

$$
t(x)=1-\omega \times\left(\frac{\text { dark_I }_{-}}{A}\right)
$$

(5) Rebuilt the defogged image using model as follow:

$$
J(x)=\frac{I(x)-A(1-t(x))}{t(x)}
$$

(6) Calculate the PSNR of images obtained from step (5) by formula (3).

(7) Set the increase step of $\omega$ (this paper is 0.1 ), increase $\omega$. Repeat the step (4), (5), (6) to get a new set of image $\mathrm{J}(\mathrm{x})$, and corresponding Peak Signal to Noise Ratio PSNR;

(8) If the growth of the signal to noise ratio is less than 0.01 or begins to decrease, branch step (9), otherwise, repeat steps (7);

(9) Output the best estimate concentration of haze $\omega$ and transmission distribution rate $t(x)$ corresponding to the defogged image.

\section{ANALYSIS OF THE SIMULATION}

Figure 1 to 2 shows the results of algorithm in the paper and the dark channel priority defogging algorithm [6].
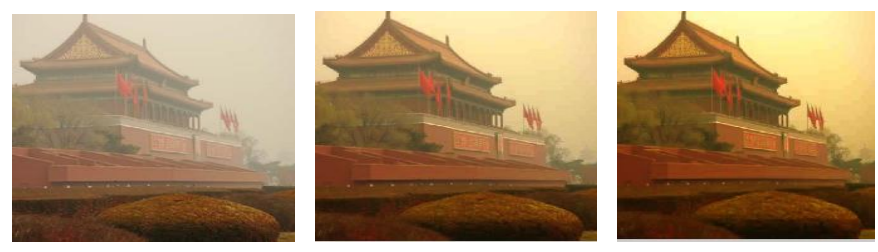

Figure 1. Result of image Tian'anmen, from left to right, its shown the source image, result using method in paper 6 and that of our method

It can clearly see from the figure, the algorithm of this paper on the visual effect is better than the original algorithm, especially the grey area in the image (close to the color of the sky), and, in this paper, the algorithm can better performance image details.
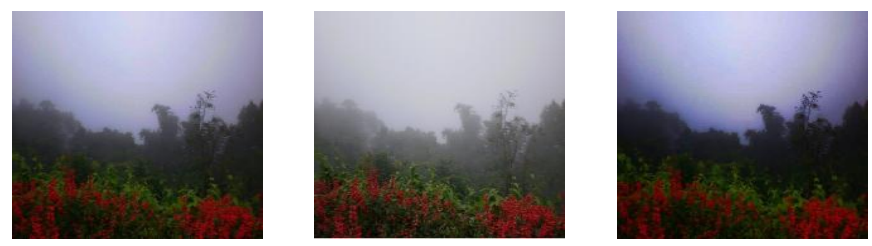

Figure 2. Result of image Mountain Emei, from left to right, its shown the source image, result using method in paper 6 and that of our method

Because the average gradient can objectively reflect the clarity of the image [15], the average gradient formula is:

$$
G=\sqrt{\frac{[I(i+1, j)-I(i, j)]^{2}+[I(i, j+1)-I(i, j)]^{2}}{2}} / m^{*} n
$$

Where, $I(i, j)$ is the image after dehazing, $m, n$ is the size of the image. In order to quantitatively illustrate the conclusion, through calculation and comparison the algorithm and grey value of the average gradient of dark channel priority algorithm in this paper. Comparison results are shown in table.

Table 1: Two algorithms to dehaze average gradient value $\mathrm{G}$

\begin{tabular}{|lcc|}
\hline Algorithm & Figure 1 & Figure2 \\
\hline Dark channel priority & 4.9853 & 3.2799 \\
This algorithm & 5.1922 & 3.5645 \\
\hline
\end{tabular}

\section{CONCLUSION}

Base on the theory of the dark channel priority, introducing the concept of peak signal to noise ratio, so that they can more accurately estimate the concentration of haze of the atomization image, and finally by calculating and comparing the average gradient of the algorithm of this paper and the original algorithm show that this algorithm can better show the details of the image. And the algorithm of this paper in the gray area of image processing visual effect is better than the original algorithm 


\section{ACKNOWLEDGMENT}

This work was supported by the Scientific Research Fund of Sichuan Provincial Artificial Intelligence Key Laboratory (2010RZ003, 2010RY003, 2011RYY02, 2011RYY04, 2012RYY05)

\section{REFERENCES}

[1] LIU Song-Tao, Wu Gang. Based on a new method of image enhancement generalized histogram equalization. Electro-optical and control, 2010,17 (3) :13-14

[2] WANG Yong research single haze-degraded image restoration method Hefei: Hefei University of Technology, 2010.

[3] Wang Chao multi-image algorithm and its application to fog Hefei: Anhui University, 2010.

[4] Zhan Xiang, Zhou Yan, an enhanced image-based haze local variance method Journal of Computer Applications, 2007, 27 (2): 510-512. 510-512.

[5] Feng Ruili, Zi-Guo Fan. Variable enhancement algorithms PCA framework model based on Retinex image.

[6] Rui Yibin, Li Peng, Sun Jintao An image to dehaze. Methods of Computer Applications. Computer Applications, 2006, 26(1): 155-156 26 (1) :155-156

[7] Ma Yunfei, where the article. Haze wavelet transform image enhancement Based Computer Applications Applications and Software 2011,28(2):71-73 2011,28 (2) :71-73
[8] Wang Hui, LIU Xiao-yang. Using atmospheric modulation transfer function recovery weather degraded image. Shenyang Institute of Aeronautical Engineering, 2006,23 (5): 94-96

[9] Dong Tao, Dong Huiying study. Degraded image restoration method based on the weather atmospheric modulation transfer function of Shenyang University of Technology, 2006, 25 (5) : 39-41

[10] Shwartz S, Shwart E, Shwart Y Y, Blind haze separation //Proc of IEEE CVPR'06.Washington: IEEE Computer Society, 2006: 1988-1991.

[11] Schechner Y Y, Narasimhan S G, Nayars K. Instant dehazing of images using polarization //Proc of IEEE CVPR'01.Washington: IEEE Computer Society, 2001: 324-331.

[12] He kaiming, Sun jian, Zhou xiaoou, Single image haze romoval using dark channel prior, Proc of IEEE CVPR'09. Washington: IEEE Computer Society, 2009: 1956-1962

[13] Levin A, Lischinski D, Weiss Y. A closed form solution to natural image matting, Pro of IEEE CVPR'06. Washington: IEEE Computer Society, 2006:61-68.

[14] Li Xiaojing. Image quality evaluation and image enhancement technology research. Nanjing University, 2008, 2-5

[15] Liu Weidong, Xu Jian, fused image quality assessment for western mapping tone painted, Geomatics Technology and Equipment, 2010, 12(2): 3-5 12 (2) : 3-5 